\title{
Triglyceride glucose index predicts coronary artery calcification better than other indices of insulin resistance in Korean adults: the Kangbuk Samsung Health Study
}

\author{
Ji-Hyun Kim, Da-Young Lee, Se Eun Park, Cheol-Young Park, Won-Young Lee, \\ Ki-Won Oh, Sung-Woo Park, Eun-Jung Rhee
}

Division of Endocrinology and Metabolism, Department of Internal Medicine, Kangbuk Samsung Hospital, Sungkyunkwan University School of Medicine, Seoul, Korea

Received: February 2, 2017

Revised: February 27, 2017

Accepted: March 14, 2017

Corresponding author:

Eun-Jung Rhee

Division of Endocrinology

and Metabolism, Department

of Internal Medicine,

Kangbuk Samsung Hospital, Sungkyunkwan University School of Medicine, 29 Saemunan-ro, Jongno-gu, Seoul 03181, Korea Tel: +82-2-2001-2485

E-mail: hongsiri@hanmail.net

This is an Open Access article distributed under the terms of the Creative Commons Attribution Non-Commercial License (http:// creativecommons.org/licenses/ by-nc/4.0/).

\section{ABSTRACT}

Purpose: Insulin resistance is one of the most important mechanisms in the development of diabetes, and it is closely related to the presence and severity of coronary heart disease. Triglyceride glucose (TyG) index is a useful marker of insulin resistance; however, few studies have investigated the relationship between TyG and subclinical atherosclerosis. Therefore, we evaluated the association of TyG and subclinical coronary atherosclerosis as measured by coronary artery calcium score (CACS).

Methods: Our study included 30,776 participants (mean age of 41 years, $80.4 \%$ male) enrolled in a health screening program, in whom CACS were measured. Homeostasis model assessment of insulin resistance (HOMA-IR), TyG index, TyG-body mass index (BMI), and TyG-waist circumference (WC) were subsequently analyzed. Indices were calculated using the following formulae: HOMA-IR = fasting insulin $(\mu \mathrm{U} / \mathrm{mL}) \times$ fasting plasma glucose $(\mathrm{FPG} ; \mathrm{mmol} / \mathrm{L}) / 22.5$; TyG index $=\mathrm{Ln}[\mathrm{TG}(\mathrm{mg} / \mathrm{dL}) \times \mathrm{FPG}(\mathrm{mg} / \mathrm{dL}) / 2]$; TyG$\mathrm{BMI}=$ TyG index $\times \mathrm{BMI}$; and TyG-WC $=$ TyG index $\times$ WC. CACS was measured using multidetector computed tomography, and the presence of coronary artery calcification (CAC) was defined by $\mathrm{CACS}>0$.

Results: The prevalence of CAC was $14.4 \%$ in the study population. Multivariate logistic regression analysis showed that participants with TyG-BMI in the highest tertile were 1.638 times more likely to have CAC after adjustment for other metabolic parameters compared with participants with TyG-BMI in the lowest tertile (odds ratio, 1.612; 95\% confidence interval, 1.465 to 1.774). The receiver operating characteristics curve for prediction of CAC showed that TyG-WC index had a higher area under the curve $(A U C=0.626)$ than other indices $\left(\mathrm{AUC}_{\text {TyG }}=0.617, \mathrm{AUC}_{\text {TуG-BмI }}=0.616, \mathrm{AUC}_{\text {номА-IR }}=0.562\right.$ ).

Conclusion: TyG index predicted CAC better than other markers of insulin resistance, and could be a useful marker for predicting subclinical atherosclerosis.

Keywords: Body mass index; Coronary artery calcification; Triglyceride glucose index; Waist circumference 


\section{PRECISION AND FUTURE MIEDICINE}

TyG index and CAC progression

\section{INTRODUCTION}

Insulin resistance is one of the most important mechanisms in the development of diabetes [1], and it is closely related to the presence and severity of cardiovascular disease [2-4]. Insulin resistance is thought to promote atherogenesis in the absence of hyperglycemia [5], and contributes to atherosclerosis by way of dyslipidemia, hypertension, and other proinflammatory conditions, as well as through the effect of impaired insulin signaling at the cellular level $[6,7]$.

Hyperinsulinemic-euglycemic clamp (HEC) is considered the gold standard for evaluating insulin resistance; however, its practical clinical use is limited by cost, labor, and ethical issues. Various indirect markers of insulin sensitivity, such as the homeostasis model assessment of insulin resistance (HOMA-IR), have been proposed that have great clinical utility [8]. In recent years, the product of triglyceride (TG) fasting plasma glucose (FPG) has been proved an alternative to HOMA-IR for estimating insulin resistance. Especially, triglyceride glucose (TyG) index has a higher sensitivity and specificity compared with HEC for estimating insulin sensitivity $[9,10]$. Thus, the superiority of TyG in identifying the insulin resistance can be demonstrated [11].

Body mass index (BMI) and waist circumference (WC) are simple, inexpensive, and noninvasive anthropometric parameters that are useful indicators of obesity and other metabolic disease. Evidence increasingly suggests that obesity is intimately associated with insulin resistance; therefore, a combination of obesity and TyG may identify insulin resistance stronger than other surrogate markers. Multiple cohort studies showed that increased insulin resistance was also associated with cardiovascular disease [12-14]. However, there are few studies focusing on the relationship between TyG and subclinical coronary atherosclerosis.

Our study aimed to investigate the association of TyG-related indices and risk of subclinical atherosclerosis in a Korean population. We also evaluated which of the TyG-related most accurately reflected the presence of coronary artery calcification (CAC) when compared with HOMA-IR.

\section{METHODS}

\section{Study participants}

This cross-sectional study was conducted using a medical health assessment program database obtained from the Health Promotion Center of Kangbuk Samsung Hospital, Sungkyunkwan University School of Medicine, Seoul, Korea. The purpose of the medical health checkup program is to promote the health of employees through a regular health checkup and to enhance early detection of existing disease.

The dataset comprised a total of 33,486 participants who participated in the program between January 2011 and December 2012. Of these participants, 2,692 were excluded from the study owing to self-reported history of ischemic heart disease $(n=419)$, ischemic stroke $(n=270)$, history of aspirin $(n=38)$ or statin $(n=1,249)$ use, and missing data $(n=869)$. Finally, we enrolled 30,776 participants into our study, of which 24,755 were men and 6,021 were women.

This study was reviewed and approved by the Institutional Review Board of Kangbuk Samsung Hospital (KBC13051), and is an accordance with the Helsinki Declaration of 1975.

\section{Anthropometric and laboratory measurements}

Height and weight measurements were performed by welltrained nurses according to standardized methods. BMI was calculated by dividing participant's weight $(\mathrm{kg})$ by the square of participant's height $(\mathrm{m})$. A single examiner measured WC in participants in the standing position by locating the halfway point between the anterior iliac crest and the lower border of the bottom rib, and then measuring circumferentially. Blood pressure was measured three times consecutively at 1-minute intervals with participants in the seated position after 5 minutes of rest, with the second and third measurements averaged.

Lifestyle habits were assessed through self-questionnaire. A smoker was defined as someone who had smoked at least five packs of cigarettes in his or her lifetime. If a participant drank more than $20 \mathrm{~g}$ of alcohol daily, he or she was classified as an alcohol drinker. Having a regular exercise habit was defined as participating in exercise of moderate intensity at least three times a week.

FPG was measured using the hexokinase method (Hitachi Modular D2400, Roche, Tokyo, Japan). The electrochemiluminescence immunoassay (Hitachi Modular E170, Roche) was used to measure fasting insulin levels. The enzymatic colorimetric test was used to assess total cholesterol (TC) and TG concentrations. Selective inhibition method and homogeneous enzymatic colorimetric assay were used to determine high density lipoprotein cholesterol (HDL-C) and low density lipoprotein cholesterol (LDL-C) levels, respectively. Insulin resistance was measured using the HOMA-IR according to the following equation: fasting insulin $(\mathrm{IU} / \mathrm{mL}) \times F P G$ $(\mathrm{mmol} / \mathrm{L}) / 22.5$ [8]. Other TyG-related markers were calculated using the following formulae: TyG index $=\mathrm{Ln}[\mathrm{TG}(\mathrm{mg} / \mathrm{dL}) \times$ 
FPG $(\mathrm{mg} / \mathrm{dL}) / 2]$; TyG-BMI = TyG index $\times$ BMI; and TyG-WC $=$ TyG index $\times$ WC [12-14].

\section{Measurement of coronary artery calcium score}

Measurement of coronary artery calcium score (CACS) was performed by 64-slice, spiral multidetector computed tomography (MDCT) scan (GE Health Care, Tokyo, Japan) using the software HEARTBEAT-CS (Philips, Cleveland, OH, USA). The 64-slice MDCT scan was performed using the following protocol: $0.625 \mathrm{~mm}$ slice thickness, $120 \mathrm{kVP}, 800$ effective mAs, and $400 \mathrm{msec}$ rotational speed.

The severity of CAC was assessed by the Agatston score [15]. The total CACS was determined by the sum of individual scores for the four major epicardial coronary arteries: left main, left anterior descending, left circumflex, and right coronary arteries. Technicians who performed the MDCT scans were blinded to all participant information.

The presence of CAC was defined by CACS $>0$. CACS was analyzed in natural logarithmic form plus 1: $\operatorname{Ln}(\mathrm{CACS}+1)$. In addition, the participants were divided into four groups according to the severity of CAC: CACS $=0 ; 0<$ CACS $\leq 100 ; 100<$
CACS $\leq 400$; and CACS $>400$.

\section{Statistical analysis}

Continuous variables were presented as the mean \pm standard deviation, and categorical variables were presented as percentages. Student t-test and chi-square test were used to compare the values of the parameters for participants with and without CACS $>0$. Analysis of variance (ANOVA) test was used to compare the mean values of parameters according to four groups divided by the severity of CAC.

Considering $85.6 \%$ of the participants had a $\mathrm{CACS}=0$, natural logarithmic transformation of CACS +1 was used for the bivariate correlation analyses, the latter performed using Pearson correlation and partial correlation analysis [16-18]. Study population was stratified into tertiles based on the following variables: HOMA-IR, TyG, TyG-BMI, and TyG-WC.

Multivariate logistic regression analysis reporting odds ratio (OR) with 95\% confidence intervals was performed to estimate the presence of CAC according to tertile group of TyG index and other metabolic parameters. Four regression models were assessed: crude (unadjusted) model, model 1 adjusted for

Table 1. Comparison of baseline characteristics between those with or without coronary artery calcification

\begin{tabular}{|c|c|c|c|c|}
\hline Characteristic & All $(n=30,776)$ & $C A C=0(n=26,356)$ & $C A C>0(n=4,420)$ & P-value \\
\hline Age (yr) & $41.2 \pm 7.3$ & $40.2 \pm 6.7$ & $47.0 \pm 8.0$ & $<0.01$ \\
\hline Male sex & $24,755(80.4)$ & $20,693(78.5)$ & $4,062(91.9)$ & $<0.01$ \\
\hline $\mathrm{SBP}(\mathrm{mm} \mathrm{Hg})$ & $113.4 \pm 12.9$ & $112.7 \pm 12.7$ & $117.6 \pm 13.0$ & $<0.01$ \\
\hline FPG (mg/dL) & $98.8 \pm 16.7$ & $97.7 \pm 14.9$ & $105.0 \pm 23.8$ & $<0.01$ \\
\hline HbAlc (\%) & $5.7 \pm 0.5$ & $5.7 \pm 0.5$ & $5.9 \pm 1.8$ & $<0.01$ \\
\hline DM & $1,785(5.8)$ & $1,116(4.2)$ & $669(15.1)$ & $<0.01$ \\
\hline $\mathrm{TC}(\mathrm{mg} / \mathrm{dL})$ & $202.4 \pm 35.7$ & $200.9 \pm 34.8$ & $211.1 \pm 39.7$ & $<0.01$ \\
\hline $\mathrm{TG}(\mathrm{mg} / \mathrm{dL})$ & $137.2 \pm 92.4$ & $133.18 \pm 87.60$ & $161.2 \pm 114.4$ & $<0.01$ \\
\hline LDL-C (mg/dL) & $129.2 \pm 32.5$ & $127.9 \pm 31.8$ & $136.9 \pm 35.1$ & $<0.01$ \\
\hline $\mathrm{HDL}-\mathrm{C}(\mathrm{mg} / \mathrm{dL})$ & $53.8 \pm 13.5$ & $54.2 \pm 13.6$ & $51.2 \pm 12.8$ & $<0.01$ \\
\hline $\mathrm{BMI}\left(\mathrm{kg} / \mathrm{cm}^{2}\right)$ & $24.3 \pm 3.2$ & $24.2 \pm 3.2$ & $25.2 \pm 3.0$ & $<0.01$ \\
\hline HOMA-IR & $1.6 \pm 1.5$ & $1.5 \pm 1.6$ & $1.8 \pm 1.4$ & $<0.01$ \\
\hline TyG index & $9.3 \pm 0.6$ & $9.3 \pm 0.6$ & $9.6 \pm 0.6$ & $<0.01$ \\
\hline TyG-BMI & $228.1 \pm 38.1$ & $226.0 \pm 37.9$ & $241.0 \pm 36.8$ & $<0.01$ \\
\hline TyG-WC & $797.9 \pm 113.0$ & $790.8 \pm 112.6$ & $840.1 \pm 106.5$ & $<0.01$ \\
\hline Alcohol consumption & $5,389(17.5)$ & 4,314 (16.4) & $1,075(24.3)$ & $<0.01$ \\
\hline Smoking & $15,225(49.5)$ & $12,768(48.4)$ & $2,782(62.9)$ & $<0.01$ \\
\hline Physical activity & 5,947 (19.3) & $5,020(19.0)$ & $927(21.0)$ & $<0.01$ \\
\hline
\end{tabular}

Values are presented as mean \pm standard deviation or number (\%).

CAC, coronary artery calcification; SBP, systolic blood pressure; FPG, fasting plasma glucose; HbAlc, glycosylated hemoglobin; DM, diabetes mellitus; TC, total cholesterol; TG, triglyceride; LDL-C, low density lipoprotein cholesterol; HDL-C, high density lipoprotein cholesterol; BMI, body mass index; HOMA-IR, homeostasis model assessment index of insulin resistance; TyG, triglyceride glucose; WC, waist circumference. 
age and sex, model 2 adjusted for age, sex, systolic blood pressure (SBP), and LDL-C, and model 3 adjusted for exercise and smoking status in addition to the parameters of model 2. Statistical significance was defined by a $\mathrm{P}<0.05$. All statistical analyses were performed with SPSS version 24.0 (IBM Co., Armonk, NY, USA).

\section{RESULTS}

\section{Baseline characteristics}

The baseline biochemical and clinical characteristics of the study subjects according to presence of CAC are shown in Table 1. Among 30,776 participants, the prevalence of CAC (CACS $>0)$ was $14.4 \%(n=4,420)$, of which $2.4 \%(n=737)$ had a CACS $>100$. The mean participant age was $41.2 \pm 7.3$ years, and participants with CAC were more likely to be older than those without CAC.

Compared with individuals without CAC, those with CAC were more likely to be male smokers with higher SBP, FPG, TC, TG, and LDL-C, and have higher metabolic parameters, including HOMA-IR, TyG, TyG-BMI, and TyG-WC (Table 1). These participants were also more likely to have lower HDL-C and

Table 2. Bivariate correlation of $\operatorname{Ln}(\mathrm{CACS}+1)$ with parameters

\begin{tabular}{lccc}
\hline Parameter & $\begin{array}{c}\text { Correlation } \\
\text { coefficient }^{\mathrm{a})}\end{array}$ & Model $1^{\text {b) }}$ & Model 2 $^{\text {c) }}$ \\
\hline Age & 0.340 & - & - \\
SBP & 0.129 & - & - \\
FBG & 0.149 & 0.072 & 0.071 \\
TC & 0.079 & 0.023 & 0.021 \\
TG & 0.091 & 0.040 & 0.039 \\
LDL-C & 0.078 & - & - \\
HDL-C & -0.065 & -0.021 & -0.020 \\
BMI & 0.098 & 0.034 & 0.033 \\
Fasting insulin & 0.029 & 0.017 & 0.018 \\
HOMA-IR & 0.062 & 0.039 & 0.039 \\
TyG index & 0.132 & 0.045 & 0.043 \\
TyG-BMI & 0.125 & 0.048 & 0.046 \\
TyG-WC & 0.138 & 0.046 & 0.045 \\
\hline
\end{tabular}

Ln (CACS+1), logarithmized form of coronary artery calcium score plus 1; SBP, systolic blood pressure; FBG, fasting blood glucose; TC, total cholesterol; TG, triglyceride; LDL-C, low density lipoprotein cholesterol; HDL-C, high density lipoprotein cholesterol; BMI, body mass index; HOMA-IR, homeostasis model assessment index of insulin resistance; TyG, triglyceride glucose; WC, waist circumference.

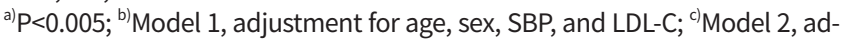
justment for age, sex, SBP, LDL-C, smoking, and regular exercise status. participate in physical activity less frequently compared with those without CAC.

\section{Correlation of CACS with variable parameters}

Bivariate correlation analysis was performed between CACS and multiple variables (Table 2). Age showed the highest correlation with CACS. After adjustment for age, sex, SBP, and LDL-C, partial correlation analyses revealed a significant correlation between all parameters and CACS. This correlation was unchanged after adjustment for smoking and physical activity. Among metabolic parameters, TyG-BMI showed the strongest correlation with CACS, followed by TyG-WC, TyG, and HOMA-IR, respectively.

\section{Comparison of subclinical atherosclerosis risk according to tertiles of metabolic parameters}

When participants were divided into tertiles based on baseline metabolic parameters, the prevalence of CAC significantly increased from the 1 st to the $3 r d$ tertile $(8.9 \%, 14.3 \%$, and 19.9\% for TyG index, $\mathrm{P}<0.001$ ) (Fig. 1). In addition, when the participants were divided into four groups according to the severity of CAC, 26,356 participants $(82.6 \%)$ had CACS $=0,3,683$ participants $(12.0 \%)$ had $0<$ CACS $\leq 100,576$ participants $(1.9 \%)$ had $100<$ CACS $\leq 400$, and 161 participants $(0.5 \%)$ had CACS $>400$ (Fig. 2). Mean values of all four parameters increased as the severity of CAC increased from CACS $=0$ to higher than 400 , when analyzed with ANOVA test (Fig. 2).

When calculating OR according to baseline metabolic parameter tertiles, the participants with TyG in the 3rd tertile were 1.488 times more likely to have $C A C>0$ and 1.644 times more likely to have CAC >100 compared with those whose

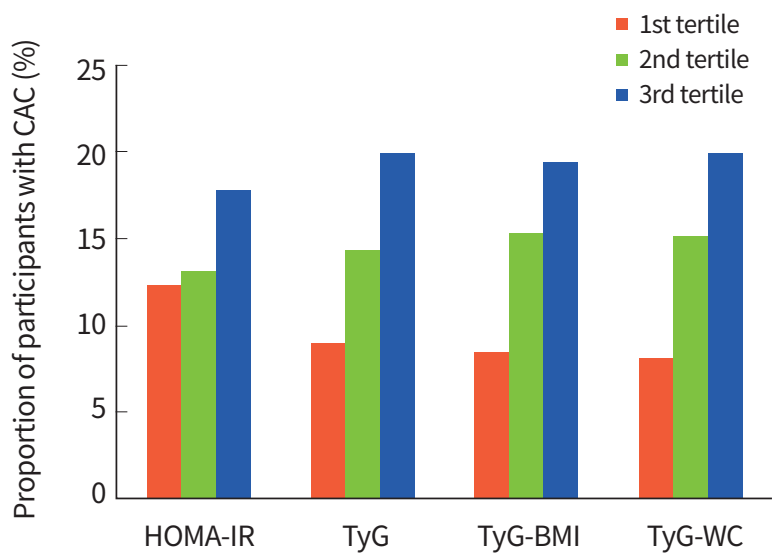

Fig. 1. Proportion of participants with coronary artery calcification (CAC) according to tertile of each parameter. HOMA-IR, homeostasis model assessment index of insulin resistance; TyG, triglyceride glucose; BMI, body mass index; WC, waist circumference. 
TyG was in the lowest tertile, even after adjustment for confounding variables (e.g., age, sex, SBP, physical activity, and

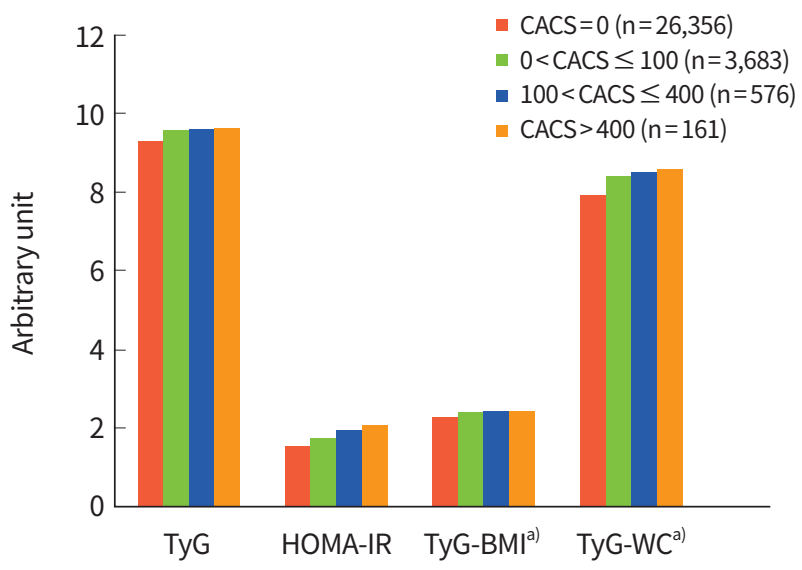

Fig. 2. Mean values of the parameters according to severity of coronary artery calcification (CAC). All mean values were significantly different according to the severity of CAC analyzed by analysis of variance test. CACS, coronary artery calcium score; TyG, triglyceride glucose; HOMA-IR, homeostasis model assessment index of insulin resistance; BMI, body mass index; WC, waist circumference. ${ }^{\text {a) }}$ Mean values were divided by 100 to be included in the same figure with other parameters. smoking status) (Table 3, Supplementary Table 1). After adjusting for traditional cardiovascular risk factors (e.g., age, sex, HTN, and LDL-C), the OR for CAC $>0$ and CAC $>100$ in participants with TyG-BMI in the 3rd tertile were 1.638 and 2.056, respectively, compared with participants whose TyG-BMI was in the lowest tertile (Table 3, Supplementary Table 1). Our results showed that TyG-related parameters were more independently associated with the presence of CAC than HOMA-IR after adjustment for other metabolic parameters.

The TyG-related metabolic parameters achieved moderate

Table 4. Areas under the receiver operating characteristic curves of each parameter for coronary artery calcification

\begin{tabular}{lc}
\hline Variable & Area under the ROC curve \\
\hline HOMA-IR & 0.562 \\
TyG index & 0.617 \\
TyG-BMI & 0.616 \\
TyG-WC & 0.626 \\
\hline
\end{tabular}

ROC, receiver operating characteristic; HOMA-IR, homeostasis model assessment index of insulin resistance; TyG, triglyceride glucose; BMI, body mass index; WC, waist circumference.

Table 3. Relative risk for coronary artery calcium score $>0$ according to tertiles of each baseline parameter

\begin{tabular}{|c|c|c|c|c|}
\hline \multirow{2}{*}{ Parameter } & \multicolumn{4}{|c|}{ OR $(95 \% \mathrm{Cl})$} \\
\hline & Unadjusted & Model $1^{\text {a) }}$ & Model $2^{\text {b) }}$ & Model $3^{c)}$ \\
\hline \multicolumn{5}{|l|}{ HOMA-IR } \\
\hline 1st tertile & 1.000 & 1.000 & 1.000 & 1.000 \\
\hline 2nd tertile & $1.078(0.993-1.170)$ & $1.138(1.040-1.245)$ & $1.035(0.945-1.134)$ & $1.037(0.946-1.136)$ \\
\hline 3rd tertile & $1.545(1.430-1.670)$ & $1.631(1.497-1.776)$ & $1.368(1.252-1.495)$ & $1.367(1.251-1.494)$ \\
\hline \multicolumn{5}{|l|}{ TyG index } \\
\hline 1st tertile & 1.000 & 1.000 & 1.000 & 1.000 \\
\hline 2nd tertile & $1.712(1.568-1.869)$ & $1.325(1.204-1.458)$ & $1.154(1.047-1.273)$ & $1.143(1.036-1.261)$ \\
\hline 3rd tertile & $2.541(2.337-2.762)$ & $1.894(1.727-2.076)$ & $1.513(1.375-1.666)$ & $1.488(1.351-1.639)$ \\
\hline \multicolumn{5}{|l|}{ TyG-BMI } \\
\hline 1st tertile & 1.000 & 1.000 & 1.000 & 1.000 \\
\hline 2nd tertile & $1.97(1.804-2.152)$ & $1.463(1.328-1.612)$ & $1.266(1.147-1.398)$ & $1.253(1.135-1.384)$ \\
\hline 3rd tertile & $2.635(2.419-2.869)$ & 2.137 (1.945-2.349) & 1.667 (1.509-1.842) & $1.638(1.482-1.810)$ \\
\hline \multicolumn{5}{|l|}{ TyG-WC } \\
\hline 1st tertile & 1.000 & 1.000 & 1.000 & 1.000 \\
\hline 2nd tertile & $2.017(1.845-2.206)$ & $1.41(1.278-1.556)$ & $1.214(1.098-1.342)$ & $1.201(1.086-1.329)$ \\
\hline 3rd tertile & $2.816(2.584-3.068)$ & $2.046(1.859-2.252)$ & $1.582(1.430-1.750)$ & $1.554(1.404-1.721)$ \\
\hline
\end{tabular}

$\mathrm{OR}$, odds ratio; $\mathrm{Cl}$, confidence interval; HOMA-IR, homeostasis model assessment index of insulin resistance; TyG, triglyceride glucose; BMI, body mass index; WC, waist circumference.

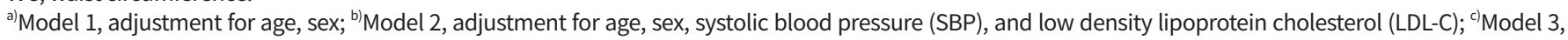
adjustment for age, sex, SBP, LDL-C, exercise, and smoking. 
prognostic performance for $\mathrm{CAC}$. The highest area under the curve (AUC) was demonstrated by TyG-WC (AUC $=0.626)$, followed by TyG (AUC=0.617), TyG-BMI (AUC=0.616), and HOMA-IR (AUC $=0.562)$ (Table 4).

\section{DISCUSSION}

In this large cohort study, TyG, TyG-BMI, and TyG-WC are more independently associated with the presence of subclinical atherosclerosis than HOMA-IR after adjustment for age, sex, and other clinical risk factors. Among these indices, TyGWC and TyG-BMI, which are associated with adiposity, showed the strongest association with subclinical atherosclerosis as measured by CACS, a marker that predicts coronary heart disease events [19]. Participants with TyG-BMI and TyG-WC in the highest tertile were approximately 1.6 times more likely to have subclinical atherosclerosis after adjustment for confounding variables compared with those who had TyG-related parameters in the lowest tertile. According to receiver operating characteristic analysis, TyG-BMI and TyG-WC were the most desirable surrogate markers of CAC after adjustment of the cofactor, among the IR indices. Therefore, our data provide evidence supporting the use of TyG-related indices for predicting early cardiovascular disease.

Previous invasive studies have reported an association between TyG index and carotid atherosclerosis. Irace et al. [20] showed that, unlike the HOMA index, the TyG index remained significantly associated with carotid atherosclerosis, even after adjustment for all traditional cardiovascular disease risk factors. A study by Tripolino et al. [21] reported that increasing insulin resistance, as assessed by TyG index, is associated with atherosclerosis-prone shear stress reduction in the common carotid artery. These findings suggested that reduced sensitivity to insulin is associated with an increase in shear stress on the arterial wall, predisposing the individual to the development of atheromatous plaques. However, few studies have evaluated the relationship between TyG index and CAC.

In a small study ( $\mathrm{n}=888$ ) performed by Lee et al. [22], individuals with TyG index in the highest tertile were 3.2 times more likely to have CAC than those with TyG index in the lowest tertile. In our study, TyG-WC and TyG-BMI were more efficient than TyG to confirm its association with CAC. Our study results are consistent with previous study findings that high TyG indices are associated with an increased risk of CAC, and that this association is stronger when factoring in patient adiposity. This suggests that particular attention should be paid toward cardiovascular disease among patients with high TyGrelated indices.

HOMA-IR reflects insulin resistance in our body, especially the insulin resistance in the liver as it indicates the ability of basal insulin to suppress hepatic glucose production in the fasted state [23]. Contrarily, an increase in plasma TG interferes with normal glucose metabolism in the muscle, resulting in reduced insulin sensitivity. Therefore, TyG index, unlike HOMA, appears to primarily reflect insulin resistance in muscle tissues [24-26]. Adipokine imbalance in the body not only promotes systemic dysfunction among metabolic processes, but also contributes to the development of cardiovascular disease [27]. Therefore, we hypothesized that TyG-related indices reflecting adiposity would be better associated with presence of CAC, which was subsequently supported by our study findings. In our study, we proved the robustness of our finding by analyzing data from a large number of participants (more than 30,000) and evaluating the prevalence of CAC by dividing each metabolic parameter level.

Our study has several limitations. Given that our study was cross-sectional in design, a cause-and-effect relationship could not be determined. A longitudinal study is warranted for further exploration of this relationship. Another limitation of our study is the lack of cultural and ethnic diversity of our study population. As there were interethnic differences in insulin sensitivity and in lipid profile, caution is advised when using a lipid surrogate for insulin resistance [28-30]. Additionally, the relationship among BMI, WC, and adiposity is influenced by ethnicity, supporting the use of ethnic-specific BMI and WC [31]. Another potential study limitation includes the retrospective collection of data, which could introduce various confounding factors. It is possible that our method of participant recruitment introduced selection bias into our study, as these participants were voluntarily recruited during general health assessments. Lastly, our study compared TyG-related indices with HOMA-IR, not the HEC; therefore, we were unable to provide supporting evidence favoring use of TyG-related indices, rather than HEC, as the gold standard measurement of insulin sensitivity. However, studies have shown that HOMA-IR is an appropriate alternative to the HEC [32], and that TyG index has superior sensitivity and specificity compared with the HEC for measuring insulin sensitivity [9]. Despite these limitations, our study has important clinical implications as it is the first to compare the relationship between CAC and various indirect measurements of insulin resistance. In conclusion, TyG index may be a useful predictor of subclinical coronary artery disease in healthy Korean individu- 
als, and is a more accurate predictor of CAC compared with HOMA-IR. It is important that physicians are aware of the increased risk of cardiovascular disease among patients with an elevated TyG-related index. Upon identification of at-risk patients, it is essential to emphasize lifestyle modification, including weight loss and excellent glycemic control, to prevent progression of cardiovascular disease. We recommend that future studies focusing on the relationship between TyG index and subclinical atherosclerosis be longitudinal in design and include diverse racial and ethnic populations, as this can affect study outcomes.

\section{CONFLICTS OF INTEREST}

No potential conflict of interest relevant to this article was reported.

\section{REFERENCES}

1. Lillioja S, Mott DM, Spraul M, Ferraro R, Foley JE, Ravussin $\mathrm{E}$, et al. Insulin resistance and insulin secretory dysfunction as precursors of non-insulin-dependent diabetes mellitus. Prospective studies of Pima Indians. N Engl J Med 1993;329:1988-92.

2. Bonora E, Formentini G, Calcaterra F, Lombardi S, Marini F, Zenari L, et al. HOMA-estimated insulin resistance is an independent predictor of cardiovascular disease in type 2 diabetic subjects: prospective data from the Verona Diabetes Complications Study. Diabetes Care 2002;25:113541.

3. Welsh P, Preiss D, Lloyd SM, de Craen AJ, Jukema JW, Westendorp RG, et al. Contrasting associations of insulin resistance with diabetes, cardiovascular disease and all-cause mortality in the elderly: PROSPER long-term follow-up. Diabetologia 2014;57:2513-20.

4. Emerging Risk Factors Collaboration, Sarwar N, Gao P, Seshasai SR, Gobin R, Kaptoge S, et al. Diabetes mellitus, fasting blood glucose concentration, and risk of vascular disease: a collaborative meta-analysis of 102 prospective studies. Lancet 2010;375:2215-22.

5. Yip J, Facchini FS, Reaven GM. Resistance to insulin-mediated glucose disposal as a predictor of cardiovascular disease. J Clin Endocrinol Metab 1998;83:2773-6.

6. Bornfeldt KE, Tabas I. Insulin resistance, hyperglycemia, and atherosclerosis. Cell Metab 2011;14:575-85.

7. Giacco F, Brownlee M. Oxidative stress and diabetic complications. Circ Res 2010;107:1058-70.
8. Matthews DR, Hosker JP, Rudenski AS, Naylor BA, Treacher DF, Turner RC. Homeostasis model assessment: insulin resistance and beta-cell function from fasting plasma glucose and insulin concentrations in man. Diabetologia 1985; 28:412-9.

9. Guerrero-Romero F, Simental-Mendia LE, Gonzalez-Ortiz M, Martinez-Abundis E, Ramos-Zavala MG, HernandezGonzalez SO, et al. The product of triglycerides and glucose, a simple measure of insulin sensitivity. Comparison with the euglycemic-hyperinsulinemic clamp. J Clin Endocrinol Metab 2010;95:3347-51.

10. Simental-Mendia LE, Rodriguez-Moran M, Guerrero-Romero F. The product of fasting glucose and triglycerides as surrogate for identifying insulin resistance in apparently healthy subjects. Metab Syndr Relat Disord 2008;6:299304.

11. Taniguchi A, Fukushima M, Sakai M, Miwa K, Makita T, Nagata I, et al. Remnant-like particle cholesterol, triglycerides, and insulin resistance in nonobese Japanese type 2 diabetic patients. Diabetes Care 2000;23:1766-9.

12. Park SH, Lee WY, Lee YS, Rhee EJ, Kim SW. The relative effects of obesity and insulin resistance on cardiovascular risk factors in nondiabetic and normotensive men. Korean J Intern Med 2004;19:75-80.

13. Fakhrzadeh H, Sharifi F, Alizadeh M, Arzaghi SM, Tajallizade-Khoob Y, Tootee A, et al. Relationship between insulin resistance and subclinical atherosclerosis in individuals with and without type 2 diabetes mellitus. J Diabetes Metab Disord 2015;15:41.

14. Bertoni AG, Wong ND, Shea S, Ma S, Liu K, Preethi S, et al. Insulin resistance, metabolic syndrome, and subclinical atherosclerosis: the Multi-Ethnic Study of Atherosclerosis (MESA). Diabetes Care 2007;30:2951-6.

15. Rumberger JA, Brundage BH, Rader DJ, Kondos G. Electron beam computed tomographic coronary calcium scanning: a review and guidelines for use in asymptomatic persons. Mayo Clin Proc 1999;74:243-52.

16. Ishiyama M, Suzuki E, Katsuda J, Murase H, Tajima Y, Horikawa $\mathrm{Y}$, et al. Associations of coronary artery calcification and carotid intima-media thickness with plasma concentrations of vascular calcification inhibitors in type 2 diabetic patients. Diabetes Res Clin Pract 2009;85:189-96.

17. Mikami S, Hamano T, Fujii N, Nagasawa Y, Isaka Y, Moriyama T, et al. Serum osteoprotegerin as a screening tool for coronary artery calcification score in diabetic pre-dialysis patients. Hypertens Res 2008;31:1163-70.

18. Han D, B OH, Gransar H, Yoon JH, Kim KJ, Kim MK, et al. 


\section{PRECISION AND FUTURE MIEDICINE}

TyG index and CAC progression

Incremental benefit of coronary artery calcium score above traditional risk factors for all-cause mortality in asymptomatic Korean adults. Circ J 2015;79:2445-51.

19. Arad Y, Spadaro LA, Goodman K, Newstein D, Guerci AD. Prediction of coronary events with electron beam computed tomography. J Am Coll Cardiol 2000;36:1253-60.

20. Irace C, Carallo C, Scavelli FB, De Franceschi MS, Esposito T, Tripolino $C$, et al. Markers of insulin resistance and carotid atherosclerosis. A comparison of the homeostasis model assessment and triglyceride glucose index. Int J Clin Pract 2013;67:665-72.

21. Tripolino C, Irace C, Scavelli FB, de Franceschi MS, Esposito T, Carallo C, et al. Triglyceride glucose index and common carotid wall shear stress. J Investig Med 2014;62:340-4.

22. Lee EY, Yang HK, Lee J, Kang B, Yang Y, Lee SH, et al. Triglyceride glucose index, a marker of insulin resistance, is associated with coronary artery stenosis in asymptomatic subjects with type 2 diabetes. Lipids Health Dis 2016; 15:155.

23. Tripathy D, Almgren P, Tuomi T, Groop L. Contribution of insulin-stimulated glucose uptake and basal hepatic insulin sensitivity to surrogate measures of insulin sensitivity. Diabetes Care 2004;27:2204-10.

24. Katsuki A, Sumida Y, Urakawa H, Gabazza EC, Murashima $\mathrm{S}$, Maruyama N, et al. Increased visceral fat and serum levels of triglyceride are associated with insulin resistance in Japanese metabolically obese, normal weight subjects with normal glucose tolerance. Diabetes Care 2003;26: 2341-4.

25. Kelley DE, Goodpaster BH. Skeletal muscle triglyceride. An aspect of regional adiposity and insulin resistance. Di- abetes Care 2001;24:933-41.

26. Pan DA, Lillioja S, Kriketos AD, Milner MR, Baur LA, Bogardus $C$, et al. Skeletal muscle triglyceride levels are inversely related to insulin action. Diabetes 1997;46:983-8.

27. Nakamura K, Fuster JJ, Walsh K. Adipokines: a link between obesity and cardiovascular disease. J Cardiol 2014;63: 250-9.

28. Haffner SM, D’Agostino R, Saad MF, Rewers M, Mykkanen L, Selby J, et al. Increased insulin resistance and insulin secretion in nondiabetic African-Americans and Hispanics compared with non-Hispanic whites. The Insulin Resistance Atherosclerosis Study. Diabetes 1996;45:742-8.

29. Hyatt TC, Phadke RP, Hunter GR, Bush NC, Munoz AJ, Gower BA. Insulin sensitivity in African-American and white women: association with inflammation. Obesity (Silver Spring) 2009;17:276-82.

30. Kim-Dorner SJ, Simpson-McKenzie CO, Poth M, Deuster PA. Psychological and physiological correlates of insulin resistance at fasting and in response to a meal in African Americans and Whites. Ethn Dis 2009;19:104-10.

31. Lear SA, Humphries KH, Kohli S, Birmingham CL. The use of BMI and waist circumference as surrogates of body fat differs by ethnicity. Obesity (Silver Spring) 2007;15:281724.

32. Bonora E, Targher G, Alberiche M, Bonadonna RC, Saggiani $\mathrm{F}$, Zenere MB, et al. Homeostasis model assessment closely mirrors the glucose clamp technique in the assessment of insulin sensitivity: studies in subjects with various degrees of glucose tolerance and insulin sensitivity. Diabetes Care 2000;23:57-63. 
PRECISION AND FUTURE MEDICINE

Ji-Hyun Kim, et al.

Supplementary Table 1. Relative risk for coronary artery calcium score $>100$ according to tertiles of each baseline parameter

\begin{tabular}{|c|c|c|c|c|}
\hline \multirow{2}{*}{ Parameter } & \multicolumn{4}{|c|}{ OR $(95 \% \mathrm{Cl})$} \\
\hline & Unadjusted & Model $1^{\text {a) }}$ & Model $2^{\text {b) }}$ & Model $3^{c)}$ \\
\hline \multicolumn{5}{|l|}{ HOMA-IR } \\
\hline 1st tertile & 1.000 & 1.000 & 1.000 & 1.000 \\
\hline 2nd tertile & $1.158(0.951-1.412)$ & $1.317(1.069-1.621)$ & $1.204(0.976-1.486)$ & $1.202(0.974-1.483)$ \\
\hline 3rd tertile & $1.812(1.512-2.172)$ & $2.052(1.693-2.487)$ & $1.745(1.432-2.126)$ & $1.734(1.423-2.114)$ \\
\hline \multicolumn{5}{|l|}{ TyG index } \\
\hline 1st tertile & 1.000 & 1.000 & 1.000 & 1.000 \\
\hline 2nd tertile & $1.541(1.250-1.901)$ & $1.197(0.960-1.493)$ & $1.09(0.871-1.364)$ & $1.076(0.860-1.348)$ \\
\hline 3rd tertile & $2.548(2.100-3.091)$ & $1.894(1.727-2.076)$ & $1.68(1.358-2.079)$ & $1.644(1.326-2.037)$ \\
\hline \multicolumn{5}{|l|}{ TyG-BMI } \\
\hline 1st tertile & 1.000 & 1.000 & 1.000 & 1.000 \\
\hline 2nd tertile & $2.000(1.612-2.483)$ & $1.529(1.217-1.920)$ & $1.376(1.091-1.735)$ & $1.355(1.074-1.709)$ \\
\hline 3rd tertile & $2.941(2.397-3.609)$ & $2.57(2.065-3.198)$ & $2.101(1.673-2.639)$ & $2.056(1.635-2.584)$ \\
\hline \multicolumn{5}{|l|}{ TyG-WC } \\
\hline 1st tertile & 1.000 & 1.000 & 1.000 & 1.000 \\
\hline 2nd tertile & $2.11(1.694-2.628)$ & $1.455(1.155-1.834)$ & $1.306(1.033-1.652)$ & $1.287(1.017-1.629)$ \\
\hline 3rd tertile & $3.134(2.546-3.859)$ & $2.299(1.841-2.870)$ & $1.863(1.479-2.346)$ & $1.823(1.445-2.300)$ \\
\hline
\end{tabular}

$\mathrm{OR}$, odds ratio; $\mathrm{Cl}$, confidence interval; HOMA-IR, homeostasis model assessment index of insulin resistance; TyG, triglyceride glucose; BMI, body mass index; WC, waist circumference.

${ }^{\text {a) }}$ Model 1 , adjustment for age and sex; ${ }^{\text {b) }}$ Model 2, adjustment for age, sex, systolic blood pressure (SBP), and low density lipoprotein cholesterol (LDL-C); ${ }^{\text {C) Model }}$ 3 , adjustment for age, sex, SBP, LDL-C, exercise, and smoking. 